\title{
IDEALS OF COMPACT OPERATORS
}

\author{
ÅSVALD LIMA and EVE OJA
}

(Received 14 September 2002; revised 24 April 2003)

Communicated by $\mathrm{G}$. Willis

\begin{abstract}
We give an example of a Banach space $X$ such that $\mathscr{K}(X, X)$ is not an ideal in $\mathscr{K}\left(X, X^{* *}\right)$. We prove that if $z^{*}$ is a weak denting point in the unit ball of $Z^{*}$ and if $X$ is a closed subspace of a Banach space $Y$, then the set of norm-preserving extensions $H B\left(x^{*} \otimes z^{*}\right) \subseteq \mathscr{L}\left(Z^{*}, Y\right)^{*}$ of a functional $x^{*} \otimes z^{*} \in(Z \otimes X)^{*}$ is equal to the set $H B\left(x^{*}\right) \otimes\left\{z^{*}\right\}$. Using this result, we show that if $X$ is an $M$-ideal in $Y$ and $Z$ is a reflexive Banach space, then $\mathscr{K}(Z, X)$ is an $M$-ideal in $\mathscr{K}(Z, Y)$ whenever $\mathscr{K}(Z, X)$ is an ideal in $\mathscr{K}(Z, Y)$. We also show that $\mathscr{K}(Z, X)$ is an ideal (respectively, an $M$-ideal) in $\mathscr{K}(Z, Y)$ for all Banach spaces $Z$ whenever $X$ is an ideal (respectively, an $M$-ideal) in $Y$ and $X^{*}$ has the compact approximation property with conjugate operators.
\end{abstract}

2000 Mathematics subject classification: primary 46B20, 46B28, 47L05.

Keywords and phrases: compact operator, ideal, $M$-ideal, approximation property.

\section{Introduction}

Let us recall that a closed subspace $F$ of a Banach space $E$ is an ideal in $E$ if $F^{\perp}$, the annihilator of $F$ in $E^{*}$, is the kernel of a norm one projection on $E^{*}$. The notion of an ideal was introduced and studied by Godefroy, Kalton, and Saphar in [8].

Let $X$ and $Y$ be Banach spaces. We denote by $\mathscr{L}(X, Y)$ the Banach space of bounded linear operators from $X$ to $Y$, and by $\mathscr{F}(X, Y), \overline{\mathscr{F}(X, Y)}, \mathscr{K}(X, Y)$, and $\mathscr{W}(X, Y)$ its subspaces of finite rank operators, approximable operators (that is, norm limits of finite rank operators), compact operators, and weakly compact operators.

In [15, Theorem 3] the following result was proved.

THEOREM 1.1. $\overline{\mathscr{F}(X, X)}$ is an ideal in $\overline{\mathscr{F}\left(X, X^{* *}\right)}$ for every Banach space $X$.

The research of the second-named author was partially supported by Estonian Science Foundation Grant 4400 and the Norwegian Academy of Science and Letters.

(C) 2004 Australian Mathematical Society 1446-7887/04 $\$$ A2.00+0.00 
In [15, page 455] it is stated as an open problem whether $\mathscr{K}(X, X)$ is an ideal in $\mathscr{K}\left(X, X^{* *}\right)$ for every Banach space $X$.

EXAMPLE 1.2. There exists a separable Banach space $X$ such that

- $\mathscr{K}\left(X, X^{* *}\right)$ is an ideal in $\mathscr{L}\left(X, X^{* *}\right)$,

- $\mathscr{K}(X, X)$ is not an ideal in $\mathscr{L}(X, X)$,

- $\mathscr{K}(X, X)$ is not an ideal in $\mathscr{K}\left(X, X^{* *}\right)$.

ProOF. Let $X=\left(\sum_{n=1}^{\infty} \oplus\left(Z^{* *},|\cdot|_{n}\right)\right)_{2}$ be the space defined (relying on the famous example due to Willis [32]) and studied by Casazza and Jarchow in [3, Theorem 1]. Recall that $Z^{* *}$ is a separable Banach space, $\mid \cdot \ln _{n}$ is an equivalent norm on $Z^{* *}$, the space $X$ fails the metric compact approximation property, but its dual space $X^{*}$ has the metric compact approximation property. Since $Z^{* *}$ is separable, the spaces $\left(Z^{* *},|\cdot|_{n}\right)$ have the Radon-Nikodým property. Thus $X$ has the Radon-Nikodým property. (The fact that the Radon-Nikodým property is preserved under $\ell_{p}$-direct sums $(1 \leq p<\infty)$ is mentioned in [6, page 219]. It can be proved following the idea of the proof in [6, pages 64-65] that a Banach space with a boundedly complete basis has the Radon-Nikodým property.)

Since $X^{*}$ has the metric compact approximation property, by a well-known result due to Johnson [12], $\mathscr{K}\left(X^{*}, X^{*}\right)$ is an ideal in $\mathscr{L}\left(X^{*}, X^{*}\right)$. Thus $\mathscr{K}\left(X, X^{* *}\right)$ is an ideal in $\mathscr{L}\left(X, X^{* *}\right)$. Since $X$ has the Radon-Nikodým property but fails to have the metric compact approximation property, by [15, Theorem 14], $\mathscr{K}(X, X)$ is not an ideal in $\mathscr{L}(X, X)$. Hence $\mathscr{K}(X, X)$ is not an ideal in $\mathscr{L}\left(X, X^{* *}\right)$. (General properties of ideals that we are using here and below in this paper are immediate from the local formulation of ideals (see Lemma 2.1, (ii), in Section 2).) If $\mathscr{K}(X, X)$ were an ideal in $\mathscr{K}\left(X, X^{* *}\right)$, then $\mathscr{K}(X, X)$ would be an ideal in $\mathscr{L}\left(X, X^{* *}\right)$ (because $\mathscr{K}\left(X, X^{* *}\right)$ is an ideal in $\mathscr{L}\left(X, X^{* *}\right)$ ) which is impossible.

In [15, page 471] it is stated as an open problem if $\mathscr{K}(X, X)$ is an ideal in $\mathscr{L}(X, X)$ whenever $\mathscr{K}\left(X^{*}, X^{*}\right)$ is an ideal in $\mathscr{L}\left(X^{*}, X^{*}\right)$. Example 1.2 also solves this problem in the negative.

Every Banach space $X$ is an ideal in its bidual $X^{* *}$ (with respect to the canonical projection of $X^{* * *}$ onto $X^{*}$ ). Let us further assume that $X$ is a closed subspace of a Banach space $Y$. In Section 2 we make a preliminary study about $\mathscr{K}(Z, X)$ being an ideal in $\mathscr{K}(Z, Y)$ for a Banach space $Z$. In Proposition 2.4 we prove that if $\mathscr{K}(Z, X)$ is an ideal in $\mathscr{K}(Z, Y)$ for some $Z \neq\{0\}$, then $X$ is an ideal in $Y$. And in Proposition 2.5 we show that if $X$ is an ideal in $Y$ and $\mathscr{K}(Z, X)$ is an ideal in $\mathscr{K}\left(Z, X^{* *}\right)$, then $\mathscr{K}(Z, X)$ is an ideal in $\mathscr{K}(Z, Y)$. We also prove that the last property is separably determined (see Theorems $2.6,2.7$, and 2.8). In particular (see 
Theorem 2.8), $\mathscr{K}(X, X)$ is an ideal in $\mathscr{K}\left(X, X^{* *}\right)$ whenever $\mathscr{K}(E, E)$ is an ideal in $\mathscr{K}\left(E, E^{* *}\right)$ for every separable ideal $E$ in $X$.

The main lemma in this paper is Lemma 3.1. This is a general result describing all norm-preserving extensions of certain important functionals on operator spaces. We use Lemma 3.1, in particular, in Theorem 3.4 to prove that if $Z$ is a reflexive Banach space, if $X$ is an $M$-ideal in $Y$, and if $\mathscr{K}(Z, X)$ is an ideal in $\mathscr{K}(Z, Y)$, then $\mathscr{K}(Z, X)$ is an $M$-ideal in $\mathscr{K}(Z, Y)$. Similar results hold for $u$-ideals (see Theorems 3.5 and 3.6).

In Example 1.2 the dual space $X^{*}$ fails to have the compact approximation property with conjugate operators (although $X^{*}$ has the metric compact approximation property). The main conclusions in this paper, Corollaries 4.7 and 4.8 in Section 4, show that $\mathscr{K}(Z, X)$ is an ideal (respectively, an $M$-ideal) in $\mathscr{K}(Z, Y)$ for all Banach spaces $Z$ whenever $X$ is an ideal (respectively, an $M$-ideal) in $Y$ and $X^{*}$ has the compact approximation property with conjugate operators. The proofs rely on results from Section 3, the description of the dual space of compact operators due to Feder and Saphar [7], and the uniform isometric version of the Davis-Figiel-Johnson-Pełczyński factorization theorem due to Lima, Nygaard, and Oja [17].

The notation we use is standard (see [21]). We consider Banach spaces over the real field $\mathbb{R}$. The closure of a set $A$ is denoted by $\bar{A}$, its linear span by span $A$, and its convex hull by conv $A$. The closed unit ball of a Banach space $E$ is denoted by $B_{E}$ and the identity operator on $E$ by $I_{E}$.

\section{Ideals of compact operators}

Let $X$ be a closed subspace of a Banach space $Y$. In this section we make a preliminary study about $\mathscr{K}(Z, X)$ being an ideal in $\mathscr{K}(Z, Y)$ for a Banach space $Z$. A first basic result, Proposition 2.4, says that if $\mathscr{K}(Z, X)$ is an ideal in $\mathscr{K}(Z, Y)$ for some Banach space $Z \neq\{0\}$, then $X$ is an ideal in $Y$. In Proposition 2.5 we show that the converse is true whenever $\mathscr{K}(Z, X)$ is an ideal in $\mathscr{K}\left(Z, X^{* *}\right)$. And we prove that the last property is separably determined (see Theorems 2.6-2.8).

In Proposition 2.10 we show that if $\mathscr{K}(Z, X)$ is an $M$-ideal (respectively, a $u$-ideal) in $\mathscr{K}(Z, Y)$ for some Banach space $Z \neq\{0\}$, then $X$ is an $M$-ideal (respectively, a $u$-ideal) in $Y$.

Let $F$ be a subspace of a Banach space $E$. A linear operator $\Phi: F^{*} \rightarrow E^{*}$ is called a Hahn-Banach extension operator if $\left(\Phi x^{*}\right)(x)=x^{*}(x)$ and $\left\|\Phi x^{*}\right\|=\left\|x^{*}\right\|$ for all $x \in F$ and all $x^{*} \in F^{*}$. The next result is well known. A proof can be found in [15].

LEMMA 2.1. Let $F$ be a closed subspace of a Banach space $E$. The following statements are equivalent. 
(i) $F$ is an ideal in $E$.

(ii) $F$ is locally 1 -complemented in $E$, that is, for every finite dimensional subspace $G$ of $E$ and for all $\varepsilon>0$, there is an operator $U: G \rightarrow F$ such that $\|U\| \leq 1+\varepsilon$ and $U x=x$ for all $x \in G \cap F$.

(iii) There exists a Hahn-Banach extension operator $\Phi: F^{*} \rightarrow E^{*}$.

In [31] (see also [11, page 282]) Werner has shown that if $X$ is an $M$-ideal in $Y$, then the injective tensor product $X \hat{\otimes}_{\epsilon} Z$ is an $M$-ideal in $Y \hat{\otimes}_{\epsilon} Z$ for any Banach space $Z$. Rao extended this to ideals in [28]. We have a short proof of this result.

Proposition 2.2. Let $X$ be an ideal in $Y$ and let $Z$ be an ideal in $W$. Then $X \hat{\otimes}_{\epsilon} Z$ is an ideal in $Y \hat{\otimes}_{\epsilon} W$.

ProOF. Let $\phi: X^{*} \rightarrow Y^{*}$ and $\psi: Z^{*} \rightarrow W^{*}$ be Hahn-Banach extension operators. Let $Q: Z^{* * *} \rightarrow Z^{*}$ be the canonical projection. We shall use the identifications $\left(X \hat{\otimes}_{\epsilon} Z\right)^{*}=I\left(X, Z^{*}\right)$ and $\left(Y \hat{\otimes}_{\epsilon} W\right)^{*}=I\left(Y, W^{*}\right)$ (see, for example, [6]). Since $\phi^{*} x=x, x \in X$, and $\psi^{*} z=z, z \in Z$, the map $\Phi: I\left(X, Z^{*}\right) \rightarrow I\left(Y, W^{*}\right)$ defined by $\Phi(T)=\left.\psi \circ Q \circ T^{* *} \circ \phi^{*}\right|_{\gamma}$ is clearly a Hahn-Banach extension operator.

Theorem 1.1 has an easy generalization.

COROLlaRY 2.3. Let $X$ be a closed subspace of a Banach space $Y$. The following statements are equivalent.

(i) $X$ is an ideal in $Y$.

(ii) $\overline{\mathscr{F}(Z, X)}$ is an ideal in $\overline{\mathscr{F}(Z, Y)}$ for all Banach spaces $Z$.

(iii) $\overline{\mathscr{F}(Z, X)}$ is an ideal in $\overline{\mathscr{F}(Z, Y)}$ for some Banach space $Z \neq\{0\}$.

In particular, $\overline{\mathscr{F}(Z, X)}$ is an ideal in $\overline{\mathscr{F}\left(Z, X^{* *}\right)}$ for all Banach spaces $X$ and $Z$.

ProOF. (i) $\Rightarrow$ (ii) is immediate from Proposition 2.2 because $\overline{\mathscr{F}(Z, X)}$ and $\overline{\mathscr{F}(Z, Y)}$ can be canonically identified with $Z^{*} \hat{\otimes}_{\epsilon} X$ and $Z^{*} \hat{\otimes}_{\epsilon} Y$.

(ii) $\Rightarrow$ (iii) is trivial.

(iii) $\Rightarrow$ (i). Suppose $\overline{\mathscr{F}(Z, X)}$ is an ideal in $\overline{\mathscr{F}(Z, Y)}$. Let $F$ be a finite dimensional subspace of $Y$. Let $z \in Z$ and $z^{*} \in Z^{*}$ be such that $\|z\|=\left\|z^{*}\right\|=z^{*}(z)=1$. Denote $G=\left\{z^{*} \otimes y: y \in F\right\} \subseteq \mathscr{F}(Z, Y)$. Let $\epsilon>0$ and let $V: G \rightarrow \overline{\mathscr{F}(Z, X)}$ be an operator such that $\|V\| \leq 1+\epsilon$ and $V(S)=S$ for all $S \in G \cap \overline{\mathscr{F}(Z, X)}$. Now define a map $U: F \rightarrow X$ by $U y=\left(V\left(z^{*} \otimes y\right)\right) z$. Then $U$ 'locally 1-complements' $X$ in $Y$.

Example 1.2 shows that the implication (i) $\Rightarrow$ (ii) of Corollary 2.3 fails if we consider compact operators instead of approximable operators. However, by the proof of the implication (iii) $\Rightarrow$ (i), we also have the similar result in the case of compact operators. 
PROPOSITION 2.4. Let $X$ be a closed subspace of a Banach space $Y$. If $\mathscr{K}(Z, X)$ is an ideal in $\mathscr{K}(Z, Y)$ for some Banach space $Z \neq\{0\}$, then $X$ is an ideal in $Y$.

PROPOSITION 2.5. Let $X$ be a closed subspace of $Y$ and assume that $\mathscr{K}(Z, X)$ is an ideal in $\mathscr{K}\left(Z, X^{* *}\right)$ for some Banach space $Z \neq\{0\}$. Then $X$ is an ideal in $Y$ if and only if $\mathscr{K}(Z, X)$ is an ideal in $\mathscr{K}(Z, Y)$.

Proof. In view of Proposition 2.4 we only need to prove the 'only if' part. Let $\phi: X^{*} \rightarrow Y^{*}$ and $\Phi: \mathscr{K}(Z, X)^{*} \rightarrow \mathscr{K}\left(Z, X^{* *}\right)^{*}$ be Hahn-Banach extension operators. Define $\Psi: \mathscr{K}(Z, X)^{*} \rightarrow \mathscr{K}(Z, Y)^{*}$ by

$$
(\Psi f)(T)=(\Phi f)\left(\left.\phi^{*}\right|_{Y} \circ T\right), \quad f \in \mathscr{K}(Z, X)^{*}, \quad T \in \mathscr{K}(Z, Y) .
$$

Then $\Psi$ is linear and $\|\Psi\| \leq 1$. Since $\phi^{*} x=x, x \in X$, we have $\left.\phi^{*}\right|_{Y} \circ T=T$ whenever $T \in \mathscr{K}(Z, X)$. Consequently, for any $T \in \mathscr{K}(Z, X)$ and any $f \in \mathscr{K}(Z, X)^{*}$, $(\Psi f)(T)=(\Phi f)(T)=f(T)$ meaning that $\Psi f$ is an extension of $f$. Hence $\Psi$ is a Hahn-Banach extension operator.

From the last proposition it follows that it is important to decide when $\mathscr{K}(Z, X)$ is an ideal in $\mathscr{K}\left(Z, X^{* *}\right)$. Example 1.2 in the Introduction shows that this is not true for all $X$ and all $Z$. If $X$ is the range of a norm one projection in $X^{* *}$, then $\mathscr{K}(Z, X)$ is an ideal in $\mathscr{K}\left(Z, X^{* *}\right)$ for all $Z$. (If $P$ is the projection, then $T \rightarrow P \circ T$ is a norm one projection from $\mathscr{K}\left(Z, X^{* *}\right)$ onto $\mathscr{K}(Z, X)$.)

We shall prove in Theorem 4.2 that $\mathscr{K}(Z, X)$ is an ideal in $\mathscr{K}\left(Z, X^{* *}\right)$ for all Banach spaces $Z$ if this is true for all separable reflexive Banach spaces $Z$.

The next three results show that the question about being an ideal, can be reduced to the case of separable Banach spaces.

THEOREM 2.6. Let $X$ and $Z$ be Banach spaces. If $\mathscr{K}(Z, E)$ is an ideal in $\mathscr{K}\left(Z, E^{* *}\right)$ for every separable ideal $E$ in $X$, then $\mathscr{K}(Z, X)$ is an ideal in $\mathscr{K}\left(Z, X^{* *}\right)$.

PROOF. Let $F \subseteq \mathscr{K}\left(Z, X^{* *}\right)$ be a finite dimensional subspace and let $\epsilon>0$. Since $G=\{T z: z \in Z, T \in F \cap \mathscr{K}(Z, X)\}$ is contained in a separable subspace of $X$, by a result of Sims and Yost [29] (see also [11, page 138]), there exists a separable ideal $E \subseteq X$ such that $G \subseteq E$. Let $\phi: E^{*} \rightarrow X^{*}$ be a Hahn-Banach extension operator. Then $\phi^{*}: X^{* *} \rightarrow E^{* *}$ and $F_{\phi}=\left\{\phi^{*} \circ T: T \in F\right\}$ is a finite dimensional subspace of $\mathscr{K}\left(Z, E^{* *}\right)$. Let $U: F_{\phi} \rightarrow \mathscr{K}(Z, E)$ be a linear operator such that $\|U\| \leq 1+\epsilon$ and $U\left(\phi^{*} \circ T\right)=\phi^{*} \circ T$ for all $T \in F \cap \mathscr{K}(Z, X)$ (note that if $T \in F \cap \mathscr{K}(Z, X)$, then $T(Z) \subseteq E$ and $\left.\phi^{*} \circ T \in \mathscr{K}(Z, E)\right)$. Now $V: F \rightarrow \mathscr{K}(Z, X)$ defined by $V(T)=U\left(\phi^{*} \circ T\right)$ 'locally 1-complements' $\mathscr{K}(Z, X)$ in $\mathscr{K}\left(Z, X^{* *}\right)$. 
REMARK 2.1. The assertion of Theorem 2.6 is not reversible: $\mathscr{K}\left(X, X^{* *}\right)$ is always an ideal in $\mathscr{K}\left(X, X^{* * * *}\right)$ (because $X^{* *}$ is the range of a norm one projection in $X^{* * * *}$ ) but, for the separable Banach space $X$ (a separable ideal in $X^{* *}$ ) described in Example 1.2, $\mathscr{K}(X, X)$ is not an ideal in $\mathscr{K}\left(X, X^{* *}\right)$.

THEOREM 2.7. Let $X, Y$, and $Z$ be Banach spaces and assume $X$ is a subspace of $Y$. Then $\mathscr{K}(Z, X)$ is an ideal in $\mathscr{K}(Z, Y)$ if and only if $\mathscr{K}(W, X)$ is an ideal in $\mathscr{K}(W, Y)$ for every separable ideal $W$ in $Z$.

Proof. Assume that $\mathscr{K}(Z, X)$ is an ideal in $\mathscr{K}(Z, Y)$ and let $W$ be an ideal in $Z$. Let $\Phi: \mathscr{K}(Z, X)^{*} \rightarrow \mathscr{K}(Z, Y)^{*}$ and $\phi: W^{*} \rightarrow Z^{*}$ be Hahn-Banach extension operators. For $f \in \mathscr{K}(W, X)^{*}$ and $S \in \mathscr{K}(Z, X)$, define $\hat{f}(S)=f\left(\left.S\right|_{W}\right)$. Finally, define an operator $\Psi: \mathscr{K}(W, X)^{*} \rightarrow \mathscr{K}(W, Y)^{*}$ by

$$
(\Psi f)(T)=(\Phi \hat{f})\left(\left.T^{* *} \circ \phi^{*}\right|_{z}\right), \quad f \in \mathscr{K}(W, X)^{*}, \quad T \in \mathscr{K}(W, Y) .
$$

Then $\Psi$ is linear and $\|\Psi\| \leq 1$. For $T \in \mathscr{K}(W, X)$, we have $\left.T^{* *} \circ \phi^{*}\right|_{Z} \in \mathscr{K}(Z, X)$ and therefore

$$
\begin{aligned}
(\Psi f)(T) & =(\Phi \hat{f})\left(\left.T^{* *} \circ \phi^{*}\right|_{Z}\right)=\hat{f}\left(\left.T^{* *} \circ \phi^{*}\right|_{Z}\right) \\
& =f\left(\left.T^{* *} \circ \phi^{*}\right|_{W}\right)=f\left(\left.T^{* *}\right|_{W}\right)=f(T) .
\end{aligned}
$$

Hence $\Psi$ is a Hahn-Banach extension operator.

Conversely, assume that $\mathscr{K}(W, X)$ is an ideal in $\mathscr{K}(W, Y)$ for every separable ideal $W$ in $Z$. Let $F \subseteq \mathscr{K}(Z, Y)$ be a finite dimensional subspace and let $\epsilon>0$. The set $\left\{T^{*} y^{*}: T \in F, y^{*} \in Y^{*}\right\}$ is separable. By a theorem due to Sims and Yost [29] (see also [11, page 138]), we can find a separable ideal $W$ in $Z$ with a Hahn-Banach extension operator $\phi: W^{*} \rightarrow Z^{*}$ such that $\left\{T^{*} y^{*}: T \in F, y^{*} \in Y^{*}\right\} \subseteq \phi\left(W^{*}\right)$. Let $i: W \rightarrow Z$ be the natural embedding. Then $i^{*}: Z^{*} \rightarrow W^{*}$ is the restriction operator and we get $I_{\phi\left(W^{*}\right)}=\left.\left(\phi \circ i^{*}\right)\right|_{\phi\left(W^{*}\right)}$.

Let $F_{W}=\{T \circ i: T \in F\} \subseteq \mathscr{K}(W, Y)$. We can find an operator $V: F_{W} \rightarrow$ $\mathscr{K}(W, X)$ with $\|V\| \leq 1+\epsilon$ such that $V(S)=S$ for every $S \in F_{W} \cap \mathscr{K}(W, X)$. Define an operator $U: F \rightarrow \mathscr{X}(Z, X)$ by $U(T)=\left.(V(T \circ i))^{* *} \circ \phi^{*}\right|_{Z}$. Then $\|U\| \leq 1+\epsilon$. For any $z \in Z, y^{*} \in Y^{*}$, and $T \in F \cap \mathscr{K}(Z, X)$ we get (since $T \circ i \in F_{W} \cap \mathscr{K}(W, X)$ and $\left.T^{*} y^{*} \in \phi\left(W^{*}\right)\right)$

$$
\begin{aligned}
y^{*}(U(T) z) & =y^{*}\left((V(T \circ i))^{* *}\left(\phi^{*} z\right)\right) \\
& =y^{*}\left(\left(T^{* *} \circ i^{* *}\right)\left(\phi^{*} z\right)\right) \\
& =y^{*}\left(\left(T^{* *} \circ i^{* *} \circ \phi^{*}\right) z\right) \\
& =\left(\left(\phi \circ i^{*} \circ T^{*}\right)\left(y^{*}\right)\right)(z) \\
& =\left(T^{*} y^{*}\right)(z)=y^{*}(T z) .
\end{aligned}
$$

Thus $U(T)=T$. Hence $\mathscr{K}(Z, X)$ is an ideal in $\mathscr{K}(Z, Y)$. 
It is clear from Theorems 2.6 and 2.7 that $\mathscr{K}(X, X)$ is an ideal in $\mathscr{K}\left(X, X^{* *}\right)$ whenever $\mathscr{K}(E, F)$ is an ideal in $\mathscr{K}\left(E, F^{* *}\right)$ for all separable ideals $E$ and $F$ in $X$. However, further developing the method of proofs of those theorems we obtain the following stronger result.

THEOREM 2.8. Let $X$ be a Banach space. If $\mathscr{K}(E, E)$ is an ideal in $\mathscr{K}\left(E, E^{* *}\right)$ for every separable ideal $E$ in $X$, then $\mathscr{K}(X, X)$ is an ideal in $\mathscr{K}\left(X, X^{* *}\right)$.

Proof. Assume that $\mathscr{K}(E, E)$ is an ideal in $\mathscr{K}\left(E, E^{* *}\right)$ for every separable ideal $E$ in $X$. Let $F \subseteq \mathscr{K}\left(X, X^{* *}\right)$ be a finite dimensional subspace and let $\epsilon>0$. Since the sets $\{T x: x \in X, T \in F \cap \mathscr{K}(X, X)\} \subseteq X$ and $\left\{T^{*} x^{*}: x^{*} \in X^{*}, T \in F\right\} \subseteq X^{*}$ are separable, we can find a separable ideal $E$ in $X$ together with a Hahn-Banach extension operator $\phi: E^{*} \rightarrow X^{*}$ so that $\{T x: x \in X, T \in F \cap \mathscr{K}(X, X)\} \subseteq E$ and $\left\{T^{*} x^{*}: x^{*} \in X^{*}, T \in F\right\} \subseteq \phi\left(E^{*}\right)$ (again we used the Sims-Yost theorem). Let $i: E \rightarrow X$ be the natural embedding.

Denote $G=\left\{\phi^{*} \circ T \circ i: T \in F\right\} \subseteq \mathscr{K}\left(E, E^{* *}\right)$. Then there exists an operator $V: G \rightarrow \mathscr{K}(E, E)$ such that $\|V\| \leq 1+\epsilon$ and $V(S)=S$ for all $S \in G \cap \mathscr{K}(E, E)$. Define $U: F \rightarrow \mathscr{K}(X, X)$ by $U(T)=\left.\left(V\left(\phi^{*} \circ T \circ i\right)\right)^{* *} \circ \phi^{*}\right|_{X}, T \in F$. Then $\|U\| \leq 1+\epsilon$. We conclude by showing that $U(T)=T$ for all $T \in F \cap \mathscr{K}(X, X)$.

Let $T \in F \cap \mathscr{K}(X, X)$. Since $T x \in E, x \in X$, we have in fact that $T \in \mathscr{K}(X, E)$. We also have that $U(T) \in \mathscr{K}(X, E)$ (this is true for all $T \in F)$. Since $T \in \mathscr{K}(X, E)$ and $\phi^{*} e=e, e \in E$, we have $\phi^{*} \circ T \circ i \in \mathscr{K}(E, E)$. Hence $V\left(\phi^{*} \circ T \circ i\right)=\phi^{*} \circ T \circ i$. For any $x \in X$ and $e^{*} \in E^{*}$, we get

$$
\begin{aligned}
e^{*}(U(T) x) & =e^{*}\left(\left(\phi^{*} \circ T \circ i\right)^{* *}\left(\phi^{*} x\right)\right)=\left(\phi^{*} x\right)\left(\left(\phi^{*} \circ T \circ i\right)^{*} e^{*}\right) \\
& =\left(\phi^{*} x\right)\left(\left(i^{*} \circ T^{*} \circ \phi\right) e^{*}\right)=\left(\left(\phi \circ i^{*} \circ T^{*} \circ \phi\right) e^{*}\right)(x) \\
& =\left(\left(\phi \circ i^{*}\right)\left(T^{*} \phi e^{*}\right)\right)(x)=\left(T^{*} \phi e^{*}\right)(x)=\left(\phi e^{*}\right)(T x)=e^{*}(T x)
\end{aligned}
$$

since $T^{*} \phi e^{*} \in \phi\left(E^{*}\right), \phi \circ i^{*}=I_{\phi\left(E^{*}\right)}$, and $T x \in E$. Thus $U(T)=T$ as desired.

REMARK 2.2. The assertion of Theorem 2.8 is not reversible: see Remark 2.1 and note that $\mathscr{K}\left(X^{* *}, X^{* *}\right)$ is always an ideal in $\mathscr{K}\left(X^{* *}, X^{* * * *}\right)$.

Some ideals have additional properties. Best known are probably $M$-ideals defined by Alfsen and Effros in [1], see also [11]. A more general type of ideals, the $u$-ideals, was first introduced by Casazza and Kalton in [4] and thoroughly studied by Godefroy, Kalton, and Saphar in [8].

Let us recall that a closed subspace $F$ of a Banach space $E$ is an $M$-ideal (respectively, a $u$-ideal) in $E$ if there exists a linear projection $P$ on $E^{*}$ with $\operatorname{ker} P=F^{\perp}$ such that $\|f\|=\|P f\|+\|f-P f\|$ for all $f \in E^{*}$ (respectively, $\left\|I_{E^{*}}-2 P\right\|=1$ ). 
LEMMA 2.9 (see $[15,8]$ ). Let $F$ be a closed subspace of a Banach space $E$. Then $F$ is an $M$-ideal (respectively, a $u$-ideal) in $E$ if and only if condition (M) (respectively, condition (u)) below is satisfied.

(M) For every finite dimensional subspace $G$ in $E$ and every $\epsilon>0$, there exists $a$ linear operator $U: G \rightarrow F$ such that $U x=x$ for all $x \in G \cap F$ and $\|U x+y-U y\| \leq$ $(1+\epsilon) \max (\|x\|,\|y\|)$ for all $x, y \in G$.

(u) For every finite dimensional subspace $G$ in $E$ and every $\epsilon>0$, there exists a linear operator $U: G \rightarrow F$ such that $U x=x$ for all $x \in G \cap F$ and $\|x-2 U x\| \leq$ $(1+\epsilon)\|x\|$ for all $x \in G$.

Proof. See [15, Theorem 4] and [8, Proposition 3.6].

In the next section we shall need the following analogue of Proposition 2.4.

PROPOSITION 2.10. Let $X$ be a closed subspace of a Banach space $Y$. If $\mathscr{K}(Z, X)$ is an $M$-ideal (respectively, a $u$-ideal) in $\mathscr{K}(Z, Y)$ for some Banach space $Z \neq\{0\}$, then $X$ is an $M$-ideal (respectively, a u-ideal) in $Y$.

PROOF. We argue as in the proof of Corollary 2.3, (iii) $\Rightarrow$ (i), but we use the local formulations of $M$-ideals and $u$-ideals from Lemma 2.9 .

\section{Hahn-Banach extension operators}

Let $X$ be a subspace of a Banach space $Y$. For each $x^{*} \in X^{*}$, let $H B\left(x^{*}\right)$ denote the set of norm-preserving extensions of $x^{*}$ to $Y$. Hahn-Banach extension operators $\Phi: X^{*} \rightarrow Y^{*}$ act as linear selection functions since $\Phi x^{*} \in H B\left(x^{*}\right)$ for all $x^{*} \in X^{*}$. This shows that if we can describe the sets $H B\left(x^{*}\right)$, then we get important information about possible Hahn-Banach extension operators.

The next lemma is fundamental for the results we obtain in this paper. It describes all norm-preserving extensions of certain important functionals on operator spaces. It also explains surprisingly well why some of those functionals have unique normpreserving extensions: the reason is that all their norm-preserving extensions must have a special form that makes them unique.

LEMMA 3.1. Let $X, Y$, and $Z$ be Banach spaces and assume that $X$ is a closed subspace of $Y$. Consider $Z \otimes X$ as a subspace of $\mathscr{L}\left(Z^{*}, Y\right)$. If $z^{*}$ is a weak $k^{*}$ denting point of $B_{Z} \cdot$ and $x^{*} \in X^{*}$, then, for $x^{*} \otimes z^{*} \in(Z \otimes X)^{*}$, the equality

$$
H B\left(x^{*} \otimes z^{*}\right)=H B\left(x^{*}\right) \otimes\left\{z^{*}\right\}
$$

holds. 
PROOF. It is clear that $H B\left(x^{*}\right) \otimes\left\{z^{*}\right\} \subseteq H B\left(x^{*} \otimes z^{*}\right)$. For the converse, let $\phi=x^{*} \otimes z^{*}$ and let $\psi \in H B(\phi)$. We may assume that $\left\|x^{*}\right\|=1$. It suffices to prove

CLAIM. $\psi \in{\overline{B_{Y^{*}} \otimes\left\{z^{*}\right\}^{*}}}^{w^{*}}$ in $\mathscr{L}\left(Z^{*}, Y\right)^{*}$.

Assume that the claim has been proved. Choose a net $\left(y_{\alpha}^{*}\right)$ in $B_{Y^{*}}$ such that $y_{\alpha}^{*} \otimes z^{*} \rightarrow \psi$ weak $^{*}$. By passing to a subnet, we may assume that $y_{\alpha}^{*} \rightarrow y^{*} \in B_{Y^{*}}$ weak* . Let $z \in Z$ satisfy $z^{*}(z)=1$. Then for any $x \in X$,

$$
x^{*}(x)=x^{*}(x) z^{*}(z)=\phi(z \otimes x)=\lim _{\alpha}\left(y_{\alpha}^{*} \otimes z^{*}\right)(z \otimes x)=y^{*}(x) z^{*}(z)=y^{*}(x),
$$

so we get that $\left.y^{*}\right|_{x}=x^{*}$. Thus $y^{*} \in H B\left(x^{*}\right)$.

For any $T \in \mathscr{L}\left(Z^{*}, Y\right)$,

$$
\psi(T)=\lim _{\alpha}\left(y_{\alpha}^{*} \otimes z^{*}\right)(T)=\lim _{\alpha} y_{\alpha}^{*}\left(T z^{*}\right)=y^{*}\left(T z^{*}\right)=\left(y^{*} \otimes z^{*}\right)(T),
$$

so $\psi=y^{*} \otimes z^{*}$.

PROOF OF THE CLAIM. Suppose for contradiction that $\psi \notin \overline{\boldsymbol{B}_{Y^{*}} \otimes\left\{z^{*}\right\}}{ }^{w^{*}}$. Then for some $T \in \mathscr{L}\left(Z^{*}, Y\right)$ with $\|T\|=1$ and some $\epsilon>0$, we get

$$
\eta(T)<\psi(T)-6 \epsilon \text { for all } \eta \in B_{Y} \cdot \otimes\left\{z^{*}\right\} .
$$

By the description of denting points due to Werner [30, Lemma 2], there exist $\delta>0$ and $z \in Z$ such that $z^{*}(z)=1,\|z\| \leq 1+\delta \epsilon$, and

$$
\left(\left\|w^{*}\right\| \leq 1 \text { and } w^{*}(z)>1-\delta\right) \quad \Rightarrow \quad\left\|w^{*}-z^{*}\right\| \leq \epsilon .
$$

Choose $x \in X$ such that $x^{*}(x)=1$ and $\|x\| \leq 1+\delta \epsilon$, and define $S=z \otimes x \in Z \otimes X$. Since $\psi(S)=1$ and $\psi \in B_{\mathscr{L}\left(Z^{*}, Y\right)^{*}}=\overline{\operatorname{conv}}^{*}\left(B_{Y^{*}} \otimes B_{Z^{*}}\right)$ (the last equality being clear from the bipolar theorem), we can find $\psi_{\epsilon} \in \operatorname{conv}\left(B_{Y^{*}} \otimes B_{Z^{*}}\right)$ such that

$$
\psi_{\epsilon}(S)>1-\delta^{2} \epsilon^{2}, \quad\left|\psi_{\epsilon}(T)-\psi(T)\right|<\epsilon .
$$

Let us write $\psi_{\epsilon}=\sum_{i=1}^{m} \lambda_{i} y_{i}^{*} \otimes z_{i}^{*}$, where $z_{i}^{*} \in B_{Z^{*}}, y_{i}^{*} \in B_{Y^{*}}, \lambda_{i}>0$, and $\sum_{i=1}^{m} \lambda_{i}=1$. We may suppose that $y_{i}^{*}(x) \geq 0$ for all $i$. Let $J=\left\{i: z_{i}^{*}(z)>1-\delta\right\}$. Then we get

$$
\begin{aligned}
1-\delta^{2} \epsilon^{2} & <\psi_{\epsilon}(S)=\sum_{i=1}^{m} \lambda_{i} y_{i}^{*}(x) z_{i}^{*}(z) \\
& =\sum_{i \in J} \lambda_{i} y_{i}^{*}(x) z_{i}^{*}(z)+\sum_{i \notin J} \lambda_{i} y_{i}^{*}(x) z_{i}^{*}(z)
\end{aligned}
$$




$$
\begin{aligned}
& \leq \sum_{i \in J} \lambda_{i}(1+\delta \epsilon)^{2}+\sum_{i \notin J} \lambda_{i}(1+\delta \epsilon)(1-\delta) \\
& =(1+\delta \epsilon)\left[(1+\delta \epsilon) \sum_{i \in J} \lambda_{i}+(1-\delta) \sum_{i \notin J} \lambda_{i}\right] \\
& =(1+\delta \epsilon)\left[1+\delta \epsilon \sum_{i \in J} \lambda_{i}-\delta \sum_{i \notin J} \lambda_{i}\right] \\
& \leq(1+\delta \epsilon)\left[1+\delta \epsilon-\delta \sum_{i \notin J} \lambda_{i}\right] .
\end{aligned}
$$

Thus $1-\delta \epsilon<1+\delta \epsilon-\delta \sum_{i \notin J} \lambda_{i}$, meaning that $\sum_{i \notin J} \lambda_{i}<2 \epsilon$. Let $\eta=\sum_{i=1}^{m} \lambda_{i} y_{i}^{*} \otimes z^{*}$. Then we get

$$
\begin{aligned}
\left\|\psi_{\epsilon}-\eta\right\| & =\left\|\sum_{i=1}^{m} \lambda_{i} y_{i}^{*} \otimes\left(z_{i}^{*}-z^{*}\right)\right\| \\
& \leq \sum_{i \in J} \lambda_{i}\left\|z_{i}^{*}-z^{*}\right\|+\sum_{i \notin J} \lambda_{i}\left\|z_{i}^{*}-z^{*}\right\|<\epsilon+4 \epsilon=5 \epsilon .
\end{aligned}
$$

But $\eta \in B_{Y \cdot} \otimes\left\{z^{*}\right\}$, so

$$
\psi_{\epsilon}(T)-5 \epsilon \leq \eta(T)<\psi(T)-6 \epsilon
$$

Thus $\epsilon<\psi(T)-\psi_{\epsilon}(T)<\epsilon$, a contradiction.

REMARK 3.1. The particular case of Lemma 3.1 with $X=Y$ is precisely Lemma 4.3 in [18] stating that $x^{*} \otimes z^{*}$ has a unique norm-preserving extension to $\mathscr{L}\left(Z^{*}, X\right)$. Therefore, the proof of Lemma 3.1 provides, in particular, a new and simpler proof to Lemma 4.3 in [18] and its earlier versions [16, Lemma 3.4], [20, Theorem 3.7], [15, Lemma 11], and [15, Lemma 12]. The uniqueness of norm-preserving extensions have been used to obtain some main results in $[2,15,16,18,20]$.

Let us point out the particular case $Y=X^{* *}$ of Lemma 3.1.

Corollary 3.2. Let $X$ and $Z$ be Banach spaces. Consider $Z \otimes X$ as a subspace of $\mathscr{L}\left(Z^{*}, X^{* *}\right)$. If $z^{*}$ is a weak denting point of $B_{Z^{*}}$ and $x^{*} \in X^{*}$, then, for $x^{*} \otimes z^{*} \in$ $(Z \otimes X)^{*}$, the equality $H B\left(x^{*} \otimes z^{*}\right)=H B\left(x^{*}\right) \otimes\left\{z^{*}\right\}$ holds.

We shall say that a closed subspace $X$ of a Banach space $Y$ has the unique ideal property in $Y$ if there is at most one ideal projection, this is, at most one norm one projection $\pi$ on $Y^{*}$ with ker $\pi=X^{\perp}$. From the relation between ideal projections and 
Hahn-Banach extension operators it is clear that $X$ has the unique extension property in $Y$ if and only if there is at most one Hahn-Banach extension operator $\phi: X^{*} \rightarrow Y^{*}$.

An obvious example of subspaces having the unique ideal property is presented by subspaces having property $U: X$ is said to have property $U$ in $Y$ if every $x^{*} \in X^{*}$ has a unique norm-preserving extension to $Y$ (this notion is due to Phelps [27]; for a recent study of such subspaces see [25] and [26]). It is well known that $M$-ideals (more generally, semi $M$-ideals and $H B$-subspaces) have property $U$ and therefore they also have the unique ideal property (for a study of $u$-ideals having property $U$ see [22]).

In the case when $Y=X^{* *}$, let us note that $\phi \in \mathscr{L}\left(X^{*}, X^{* * *}\right)$ is a Hahn-Banach extension operator if and only if $\left.\phi^{*}\right|_{X^{*}} \in \mathscr{L}\left(X^{* *}, X^{* *}\right)$ has norm one and $\left.\phi^{*}\right|_{X}=I_{X}$. Thus the unique ideal property of $X$ in $X^{* *}$ is the same as the unique extension property of $X$ introduced and deeply studied by Godefroy and Saphar in [9] (using the term ' $X$ is uniquely decomposed') and [10]. Let us recall that $X$ is said to have the unique extension property if the only operator $T \in \mathscr{L}\left(X^{* *}, X^{* *}\right)$ such that $\|T\| \leq 1$ and $\left.T\right|_{X}=I_{X}$ is $T=I_{X} \cdots$

In particular, the following Banach spaces have the unique extension property (see [10]): spaces which have property $U$ in their bidual (Hahn-Banach smooth spaces), those with a Fréchet-differentiable norm, separable polyhedral Lindenstrauss spaces, spaces of compact operators $\mathscr{K}(Z, X)$ for reflexive $Z$ and $X$.

COROLlaRY 3.3. Let $X, Y$, and $Z \neq\{0\}$ be Banach spaces and assume that $X$ is a subspace of $Y$ having the unique ideal property in $Y$. If $\mathscr{K}\left(Z^{*}, X\right)$ is an ideal in $\mathscr{K}\left(Z^{*}, Y\right)$ with an ideal projection $P$, then $X$ is an ideal in $Y$ and its unique ideal projection $\pi: Y^{*} \rightarrow Y^{*}$ satisfies, for all weak ${ }^{*}$ denting points $z^{*} \in B_{Z^{*}}$ and all $y^{*} \in Y^{*}$, the equality $P\left(y^{*} \otimes z^{*}\right)=\left(\pi y^{*}\right) \otimes z^{*}$.

Proof. Proposition 2.4 yields that $X$ is an ideal in $Y$. Let $\pi: Y^{*} \rightarrow Y^{*}$ denote its unique ideal projection and let $\phi: X^{*} \rightarrow Y^{*}$ be the unique Hahn-Banach extension operator. Then $\pi=\phi j^{*}$, where $j: X \rightarrow Y$ is the natural embedding.

Let $\Phi: \mathscr{K}\left(Z^{*}, X\right)^{*} \rightarrow \mathscr{K}\left(Z^{*}, Y\right)^{*}$ be a Hahn-Banach extension operator satisfying

$$
P f=\Phi\left(\left.f\right|_{\mathscr{K}\left(Z^{*}, X\right)}\right), \quad f \in \mathscr{K}\left(Z^{*}, Y\right)^{*} .
$$

For any weak ${ }^{*}$ denting point $z^{*}$ of $B_{Z^{*}}$, by Lemma 3.1, we have

$$
\Phi\left(x^{*} \otimes z^{*}\right) \in H B\left(x^{*}\right) \otimes\left\{z^{*}\right\}, \quad x^{*} \in X^{*} .
$$

By the linearity of $\Phi$, it is straightforward that the map $\phi_{z^{*}}: X^{*} \rightarrow Y^{*}$ defined by

$$
\Phi\left(x^{*} \otimes z^{*}\right)=\left(\phi_{z^{*}} x^{*}\right) \otimes z^{*}, \quad \phi_{z^{*}} x^{*} \in H B\left(x^{*}\right), \quad x^{*} \in X^{*},
$$


is linear and therefore it is a Hahn-Banach extension operator. Thus $\phi_{z^{*}}=\phi$. And for any $y^{*} \in Y^{*}$ we get

$$
P\left(y^{*} \otimes z^{*}\right)=\Phi\left(\left(j^{*} y^{*}\right) \otimes z^{*}\right)=\left(\phi j^{*} y^{*}\right) \otimes z^{*}=\left(\pi y^{*}\right) \otimes z^{*}
$$

as desired.

In [31] Werner proved that if $X$ is an $M$-ideal in $Y$ and $Z$ is a Banach space and if $Z^{*}$ or $Y$ has the approximation property, then $\mathscr{K}(Z, X)$ is an $M$-ideal in $\mathscr{K}(Z, Y)$. The next result shows that, for reflexive $Z$, if $\mathscr{K}(Z, X)$ is an ideal in $\mathscr{K}(Z, Y)$, then it is already an $M$-ideal without any requirement about the approximation property.

THEOREM 3.4. Let $X$ be a closed subspace of a Banach space $Y$ and let $Z \neq\{0\}$ be a reflexive Banach space. Then $\mathscr{K}(Z, X)$ is an $M$-ideal in $\mathscr{K}(Z, Y)$ if and only if $X$ is an $M$-ideal in $Y$ and $\mathscr{K}(Z, X)$ is an ideal in $\mathscr{K}(Z, Y)$.

Proof. If $\mathscr{K}(Z, X)$ is an $M$-ideal in $\mathscr{K}(Z, Y)$, then $X$ is an $M$-ideal in $Y$ by Proposition 2.10.

Assume that $X$ is an $M$-ideal in $Y$ and $\mathscr{K}(Z, X)$ is an ideal in $\mathscr{K}(Z, Y)$. Let $P$ be a norm one projection on $\mathscr{K}(Z, Y)^{*}$ with $\operatorname{ker} P=\mathscr{K}(Z, X)^{\perp}$. Let $\pi$ denote an $M$-ideal projection on $Y^{*}$ with $\operatorname{ker} \pi=X^{\perp}$. By the uniqueness of Hahn-Banach extensions in the case of $M$-ideals, $\pi$ is a unique ideal projection. Hence, for all $y^{*} \in Y^{*}$ and all weak* denting points $z \in B_{Z}$, by Corollary 3.3 ,

$$
P\left(y^{*} \otimes z\right)=\left(\pi y^{*}\right) \otimes z .
$$

Since $Z=\overline{\operatorname{span}}\left(w^{*}\right.$-dent $\left.B_{Z}\right)$, we get that

$$
P\left(y^{*} \otimes z\right)=\left(\pi y^{*}\right) \otimes z, \quad y^{*} \in Y^{*}, z \in Z .
$$

Consider now any $f \in \mathscr{K}(Z, Y)^{*}$. It suffices to prove that

$$
\|P f\|+\|f-P f\| \leq\|f\| .
$$

By the description of $\mathscr{K}(Z, Y)^{*}$ due to Feder and Saphar [7, Theorem 1] (here we use once more that $Z$ is reflexive), there exists an element $u$ in the projective tensor product $Y^{*} \hat{\otimes}_{\pi} Z$ such that

$$
f(T)=\operatorname{trace}(T u), \quad T \in \mathscr{K}(Z, Y),
$$

and $\|f\|=\|u\|_{\pi}$. For any $\epsilon>0$, let $u$ be represented as $u=\sum_{n=1}^{\infty} y_{n}^{*} \otimes z_{n}$ so that

$$
\|u\|_{\pi}+\epsilon \geq \sum_{n=1}^{\infty}\left\|y_{n}^{*}\right\|\left\|z_{n}\right\| .
$$


Define $g \in \mathscr{K}(Z, Y)^{*}$ by

$$
g(T)=\sum_{n=1}^{\infty}\left(\left(\pi y_{n}^{*} \otimes z_{n}\right)(T)\right), \quad T \in \mathscr{K}(Z, Y) .
$$

Since

$$
f(T)=\sum_{n=1}^{\infty}\left(\left(y_{n}^{*} \otimes z_{n}\right)(T)\right), \quad T \in \mathscr{K}(Z, Y),
$$

it is clear that $g=P f$ and

$$
(f-g)(T)=\sum_{n=1}^{\infty}\left(\left(\left(y_{n}^{*}-\pi y_{n}^{*}\right) \otimes z_{n}\right)(T)\right), \quad T \in \mathscr{K}(Z, Y) .
$$

Therefore,

$$
\begin{aligned}
\|f\|+\epsilon & \geq \sum_{n=1}^{\infty}\left\|y_{n}^{*}\right\|\left\|z_{n}\right\|=\sum_{n=1}^{\infty}\left\|\pi y_{n}^{*}\right\|\left\|z_{n}\right\|+\sum_{n=1}^{\infty}\left\|y_{n}^{*}-\pi y_{n}^{*}\right\|\left\|z_{n}\right\| \\
& \geq\|g\|+\|f-g\|=\|P f\|+\|f-P f\|
\end{aligned}
$$

as desired.

A closed subspace $F$ of a Banach space $E$ is called a semi $M$-ideal (see [14] or [11, page 43]) if there is a (nonlinear) projection $P$ from $E^{*}$ onto $F^{\perp}$ such that $P(\lambda f+P g)=\lambda P f+P g$ and $\|f\|=\|P f\|+\|f-P f\|$ for all $f, g \in E^{*}$ and all scalars $\lambda$.

REMARK 3.2. Theorem 3.4 remains true if one replaces ' $X$ is an $M$-ideal in $Y$ ' by the weaker condition ' $X$ is a semi $M$-ideal in $Y$ '. This is clear from the fact that $X$ is an $M$-ideal in $Y$ if and only if $X$ is an ideal in $Y$ and $X$ is a semi $M$-ideal in $Y$ (see, for example, [11, page 43]). In particular, $X$ is an $M$-ideal in its bidual $X^{* *}$ whenever $X$ is a semi $M$-ideal in $X^{* *}$. We do not know whether $\mathscr{K}(Z, X)$ is an $M$-ideal in $\mathscr{K}\left(Z, X^{* *}\right)$ whenever $\mathscr{K}(Z, X)$ is a semi $M$-ideal in $\mathscr{K}\left(Z, X^{* *}\right)$.

The method of proof of Theorem 3.4 enables us to extend the theorem from $M$ ideals to more general classes of ideals (for example, to ideals $F$ in $E$ with respect to an ideal projection $P$ satisfying $\|a f+b P f\|+c\|P f\| \leq\|f\|$ for given numbers $a, b, c$, and for all $f \in E^{*}$; these ideals were recently studied in [23] and [24]) under the assumption that $X$ has the unique ideal property in $Y$. The corresponding result on $u$-ideals reads as follows.

THEOREM 3.5. Let $X$ be a closed subspace of a Banach space $Y$ having the unique ideal property in $Y$ and let $Z \neq\{0\}$ be a reflexive Banach space. Then $\mathscr{K}(Z, X)$ is 
a u-ideal in $\mathscr{K}(Z, Y)$ if and only if $X$ is a $u$-ideal in $Y$ and $\mathscr{K}(Z, X)$ is an ideal in $\mathscr{K}(Z, Y)$.

A closed subspace $F$ of a Banach space $E$ is called a strict $u$-ideal in $E$ if there exists a linear projection $P$ on $E^{*}$ with $\operatorname{ker} P=F^{\perp}$ such that $\left\|I_{E^{*}}-2 P\right\|=1$ and the range $\operatorname{ran} P$ is a norming subspace of $E^{*}$. This notion was introduced and deeply studied by Godefroy, Kalton, and Saphar [8].

THEOREM 3.6. Let $X$ be either a separable Banach space or a Banach space containing no copy of $\ell_{1}$. If $X$ is a strict $u$-ideal in $X^{* *}$ and $\mathscr{K}(Z, X)$ is an ideal in $\mathscr{K}\left(Z, X^{* *}\right)$ for a reflexive Banach space $Z$, then $\mathscr{K}(Z, X)$ is a strict u-ideal in $\mathscr{K}\left(Z, X^{* *}\right)$.

PROOF. Let $\pi: X^{* * *} \rightarrow X^{* * *}$ be the projection from the definition of a strict $u$-ideal and let $P$ denote the ideal projection on $\mathscr{K}\left(Z, X^{* *}\right)^{*}$. It follows from [8, Propositions 5.2 and 2.7] that $X^{*}$ does not contain any proper norming closed subspace. But then $X$ has the unique extension property (see [10, Proposition 2.5]) and we can apply Theorem 3.5 to conclude that $\mathscr{K}(Z, X)$ is a $u$-ideal in $\mathscr{K}\left(Z, X^{* *}\right)$. Moreover, the proof of Theorem 3.4 shows that $P$ is the desired $u$-ideal projection and

$$
P\left(x^{* * *} \otimes z\right)=\left(\pi x^{* * *}\right) \otimes z, \quad x^{* * *} \in X^{* * *}, z \in Z .
$$

In view of the last equality ran $P$ contains the functionals $x^{* * *} \otimes z$ with $x^{* * *} \in \operatorname{ran} \pi$ and $z \in Z$. But these functionals give the norm of any $T \in \mathscr{K}\left(Z, X^{* *}\right)$ (by $\|T\|=$ $\left.\sup \left\{\left|x^{* * *}(T z)\right|: x^{* * *} \in B_{\text {ran } \pi}, z \in B_{Z}\right\}\right)$ because $\operatorname{ran} \pi$ is a norming subspace (for $X^{* *}$ ) in $X^{* * *}$ (in fact, $\operatorname{ran} \pi=X^{*}($ see [8])).

\section{Ideals of compact operators and the compact approximation property}

Let $X$ be a closed subspace of a Banach space $Y$. In this section we shall prove that $\mathscr{K}(Z, X)$ is an ideal (respectively, an $M$-ideal) in $\mathscr{K}(Z, Y)$ for all Banach spaces $Z$ whenever $X$ is an ideal (respectively, an $M$-ideal) in $Y$ and $X^{*}$ has the compact approximation property with conjugate operators. We begin by showing that if results about $\mathscr{K}(Z, X)$ being an ideal (from a given class of ideals) in $\mathscr{K}(Z, Y)$ or $\mathscr{W}(Z, Y)$ are true for all reflexive Banach spaces $Z$, then they are true for all Banach spaces $Z$. The method of proof is based on the following version of a factorization result for weakly compact operators by Lima, Nygaard, and Oja in [17, Corollary 2.4].

LEMMA 4.1. Let $Y$ and $Z$ be Banach spaces and let $G$ be a finite dimensional subspace of $\mathscr{W}(Z, Y)$. Then there exist a reflexive Banach space $W$, a norm one operator $J: Z \rightarrow W$, and a linear isometry $\Phi: G \rightarrow \mathscr{W}(W, Y)$ such that $T=$ 
$\Phi(T) \circ J$ and $\overline{\operatorname{ran} T}=\overline{\operatorname{ran} \Phi(T)}$ in $Y$ for all $T \in G$. Moreover, $T$ is compact if and only if $\Phi(T)$ is compact. In particular, if $G \subseteq \mathscr{K}(Z, Y)$, then $W$ is separable.

Proof. Applying [17, Theorem 2.3], as in the proof of [17, Corollary 2.4], we can find a reflexive Banach space $W$, a norm one operator $I: W^{*} \rightarrow Z^{*}$, and a linear isometry $\Phi: G \rightarrow \mathscr{W}(W, Y)$ so that $\overline{\operatorname{ran} I^{*}}=W, T^{* *}=\Phi(T) \circ I^{*}$ for all $T \in G$, and $T$ is compact if and only if $\Phi(T)$ is compact. As in [17, Corollary 2.4], putting $J=\left.I^{*}\right|_{Z}: Z \rightarrow W$, we have $\|J\|=1$ and $T=\Phi(T) \circ J$ for all $T \in G$. Clearly $\operatorname{ran} T \subseteq \operatorname{ran} \Phi(T)$. On the other hand,

$$
\begin{aligned}
\operatorname{ran} \Phi(T) & =(\Phi(T))(W)=(\Phi(T))\left(\overline{I^{*}\left(Z^{* *}\right)}\right) \\
& \subseteq \overline{(\Phi(T))\left(I^{*}\left(Z^{* *}\right)\right)}=\overline{\operatorname{ran} T^{* *} \subseteq \overline{\operatorname{ran} T}}
\end{aligned}
$$

because $T$ is weakly compact. Hence $\overline{\operatorname{ran} T}=\overline{\operatorname{ran} \Phi(T)}$.

In particular, if $G \subseteq \mathscr{K}(Z, Y)$, then by the proof of [17, Theorem 2.3] and [5, Lemma $1,(\mathrm{xi})] W^{*}$ is separable, hence $W$ is also separable.

THEOREM 4.2. Let $X$ be a closed subspace of a Banach space $Y$. Then $\mathscr{K}(Z, X)$ is an ideal in $\mathscr{K}(Z, Y)$ for all Banach spaces $Z$ if and only if $\mathscr{K}(W, X)$ is an ideal in $\mathscr{K}(W, Y)$ for all separable reflexive Banach spaces $W$.

PROOF. The proof is similar to the proof of [17, Theorem 3.1]. Let $\mathscr{K}(W, X)$ be an ideal in $\mathscr{K}(W, Y)$ for all separable reflexive spaces $W$. For a Banach space $Z$, let $G$ be a finite dimensional subspace of $\mathscr{K}(Z, Y)$ and let $\epsilon>0$. By Lemma 4.1, we can find a separable reflexive space $W$, a norm one operator $J: Z \rightarrow W$, and a linear isometry $\Phi$ mapping $G$ into $\mathscr{K}(W, Y)$ such that $T=\Phi(T) \circ J$ for all $T \in G$. If $U: \Phi(G) \rightarrow \mathscr{K}(W, X)$ is an operator from the local formulation of the notion of an ideal (see Lemma 2.1), then the operator $V: G \rightarrow \mathscr{K}(Z, X)$ defined by $V(T)=U(\Phi(T)) \circ J, T \in G$, has the same local properties as $U$. In particular, if $T \in G \cap \mathscr{K}(Z, X)$, then $\Phi(T) \in \mathscr{K}(W, X)$ because $\operatorname{ran} \Phi(T) \subseteq \overline{\operatorname{ran} T} \subseteq X$. Hence $V(T)=\Phi(T) \circ J=T$. Thus, $\mathscr{K}(Z, X)$ is an ideal in $\mathscr{K}(Z, Y)$.

A similar result is true for special classes of (semi) ideals.

THEOREM 4.3. Let $X$ be a closed subspace of a Banach space $Y$. Then $\mathscr{K}(Z, X)$ is an $M$-ideal (respectively, a $u$-ideal or a semi $M$-ideal) in $\mathscr{K}(Z, Y)$ for all Banach spaces $Z$ if and only if $\mathscr{K}(W, X)$ is an $M$-ideal (respectively, a $u$-ideal or a semi $M$-ideal $)$ in $\mathscr{K}(W, Y)$ for all separable reflexive Banach spaces $W$.

PROOF. The proof for $M$-ideals and $u$-ideals is similar to the proof of Theorem 4.2. Instead of the local formulation of ideals, it uses the local formulations of $M$-ideals and $u$-ideals from Lemma 2.9 . 
The proof for semi $M$-ideals will use the following characterization due to Lima [14]: a closed subspace $F$ of a Banach space $E$ is a semi $M$-ideal in $E$ if and only if for all $x \in B_{E}$, all $y \in B_{F}$, and all $\epsilon>0$, there exists $z \in F$ satisfying

$$
\|x \pm y-z\| \leq 1+\epsilon .
$$

Let $\mathscr{K}(W, X)$ be a semi $M$-ideal in $\mathscr{K}(W, Y)$ for all separable reflexive Banach spaces $W$. For a Banach space $Z$, let $T \in B_{\mathscr{X}(Z, Y)}, S \in B_{\mathscr{X}(Z, X)}$, and $\epsilon>0$. Put $G=\operatorname{span}\{S, T\} \subseteq \mathscr{K}(Z, Y)$ and let $W, J$, and $\Phi$ be as in Lemma 4.1. Note that $W$ is separable and $\Phi(S) \in \mathscr{K}(W, X)$. Since $\mathscr{K}(W, X)$ is a semi $M$-ideal in $\mathscr{K}(W, Y)$, there exists $U \in \mathscr{K}(W, X)$ such that $\|\Phi(T) \pm \Phi(S)-U\| \leq 1+\epsilon$. But then $\|T \pm S-U \circ J\| \leq 1+\epsilon$, and $U \circ J \in \mathscr{K}(Z, X)$. This shows that $\mathscr{K}(Z, X)$ is a semi $M$-ideal in $\mathscr{K}(Z, Y)$.

By the same reasoning as in the proofs of Theorem 4.2 and Theorem 4.3, we can prove the following result.

THEOREM 4.4. Let $X$ be a closed subspace of a Banach space $Y$. Then $\mathscr{K}(Z, X)$ is an ideal (respectively, an $M$-ideal, a u-ideal, or a semi $M$-ideal) in $\mathscr{W}(Z, Y)$ for all Banach spaces $Z$ if and only if $\mathscr{K}(W, X)$ is an ideal (respectively, an $M$-ideal, a $u$-ideal, or a semi $M$-ideal $)$ in $\mathscr{W}(W, Y)$ for all reflexive Banach spaces $W$.

REMARK 4.1. The particular case of Theorem 4.4 for ideals and for $X=Y$ was proved in [17, Theorem 3.1].

Let us point out the following quite surprising observation.

COROLLARY 4.5. Let $X$ be a semi $M$-ideal (respectively, a $u$-ideal having the unique ideal property) in a Banach space $Y$. If $\mathscr{K}(W, X)$ is an ideal in $\mathscr{K}(W, Y)$ for all separable reflexive Banach spaces $W$, then $\mathscr{K}(Z, X)$ is an $M$-ideal (respectively, a $u$-ideal) in $\mathscr{K}(Z, Y)$ for all Banach spaces $Z$.

PROOF. The proof is immediate from Theorem 3.4 together with Remark 3.2 (respectively, Theorem 3.5) and Theorem 4.3.

We conclude by showing that Corollary 4.5 applies if $X^{*}$ has the compact approximation property with conjugate operators, that is, there exists a net $\left(K_{\alpha}\right)$ in $\mathscr{K}(X, X)$ such that $\left(K_{\alpha}^{*}\right)$ converges to $I_{X}$. uniformly on compact subsets of $X^{*}$.

THEOREM 4.6. Let $X$ be an ideal in a Banach space $Y$ with an ideal projection $\pi$ and let $Z$ be a reflexive Banach space. If $X^{*}$ has the compact approximation property with conjugate operators, then $\mathscr{K}(Z, X)$ is an ideal in $\mathscr{L}(Z, Y)$ with an ideal projection $P$ satisfying $P\left(y^{*} \otimes z\right)=\left(\pi y^{*}\right) \otimes z$ for all $y^{*} \in Y^{*}$ and all $z \in Z$. 
Proof. Let $\phi: X^{*} \rightarrow Y^{*}$ be a Hahn-Banach extension operator satisfying $\pi=$ $\phi j^{*}$, where $j: X \rightarrow Y$ is the natural embedding. We shall use the description of $\mathscr{K}(Z, X)^{*}$ due to Feder and Saphar [7, Theorem 1] (we can use it because $Z$ is reflexive). For any $g \in \mathscr{K}(Z, X)^{*}$, there exists $u \in X^{*} \hat{\otimes}_{\pi} Z$ such that

$$
g(S)=\operatorname{trace}(S u), \quad S \in \mathscr{K}(Z, X),
$$

and $\|g\|=\|u\|_{\pi}$. Let this $u=\sum_{n=1}^{\infty} x_{n}^{*} \otimes z_{n}$ with $\left\|x_{n}^{*}\right\| \rightarrow 0$ and $\sum_{n=1}^{\infty}\left\|z_{n}\right\|<\infty$. We assume that a net $\left(K_{\alpha}^{*}\right)$ with $K_{\alpha} \in \mathscr{K}(X, X)$ converges to $I_{X}$. uniformly on compact subsets of $X^{*}$. If $T \in \mathscr{L}(Z, Y)$, then $\left.K_{\alpha}^{* *} \circ \phi^{*} \circ T^{* *}\right|_{Z} \in \mathscr{K}(Z, X)$ and

$$
\begin{aligned}
\mid \operatorname{trace}( & T(\phi u))-g\left(K_{\alpha}^{* *} \circ \phi^{*} \circ T^{* *} \mid z\right) \mid \\
= & \left|\operatorname{trace}(T(\phi u))-\operatorname{trace}\left(K_{\alpha}^{* *} \circ \phi^{*} \circ T^{* *} \mid z\right) u\right| \\
= & \left|\sum_{n=1}^{\infty}\left(\phi x_{n}^{*}\right)\left(T z_{n}\right)-\sum_{n=1}^{\infty} x_{n}^{*}\left(K_{\alpha}^{* *} \phi^{*} T z_{n}\right)\right| \\
= & \left|\sum_{n=1}^{\infty}\left(\phi^{*} T z_{n}\right)\left(x_{n}^{*}-K_{\alpha}^{*} x_{n}^{*}\right)\right| \\
& \leq \sup _{n}\left\|\left(I_{X^{*}}-K_{\alpha}^{*}\right)\left(x_{n}^{*}\right)\right\|\|T\| \sum_{n=1}^{\infty}\left\|z_{n}\right\| \underset{\alpha}{\rightarrow} 0
\end{aligned}
$$

because $\left\{0, x_{1}^{*}, x_{2}^{*}, \ldots\right\}$ is a compact subset of $X^{*}$.

Let $\Phi: \mathscr{K}(Z, X)^{*} \rightarrow \mathscr{L}(Z, Y)^{*}$ be defined by

$$
\begin{aligned}
(\Phi g)(T) & =\lim _{\alpha} g\left(\left.K_{\alpha}^{* *} \circ \phi^{*} \circ T^{* *}\right|_{Z}\right) \\
& =\operatorname{trace}(T(\phi u)), \quad g \in \mathscr{K}(Z, X)^{*}, T \in \mathscr{L}(Z, Y) .
\end{aligned}
$$

The existence of the limit implies the linearity of $\Phi g$ for all $g \in \mathscr{K}(Z, X)^{*}$ and of $\Phi$. Moreover, $\|\Phi g\| \leq\|g\|$ for all $g \in \mathscr{K}(Z, X)^{*}$ because

$$
|\operatorname{trace}(T(\phi u))| \leq\|T(\phi u)\|_{\pi} \leq\|T\|\|\phi u\|_{\pi} \leq\|T\|\|\phi\|\|u\|_{\pi}=\|T\|\|g\| .
$$

Since, for any $g \in \mathscr{K}(Z, X)^{*}$ and $S \in \mathscr{K}(Z, X)$, we have

$$
\begin{gathered}
(\Phi g)(S)=\operatorname{trace}(S(\phi u))=\operatorname{trace}\left(\sum_{n=1}^{\infty} \phi x_{n}^{*} \otimes S z_{n}\right) \\
=\sum_{n=1}^{\infty} x_{n}^{*}\left(S z_{n}\right)=\operatorname{trace}(S u)=g(S),
\end{gathered}
$$

meaning that $\Phi g$ extends $g$, we conclude that $\Phi$ is a Hahn-Banach extension operator from $\mathscr{K}(Z, X)^{*}$ to $\mathscr{L}(Z, Y)^{*}$. Thus, $\mathscr{K}(Z, X)$ is an ideal in $\mathscr{L}(Z, Y)$. 
Let $P$ be the ideal projection on $\mathscr{L}(Z, Y)^{*}$ defined by $\Phi$, that is,

$$
P f=\Phi\left(\left.f\right|_{\mathscr{X}(Z, X)}\right), \quad f \in \mathscr{L}(Z, Y)^{*}
$$

Considering $x^{*} \otimes z \in \mathscr{K}(Z, X)^{*}$ with $x^{*} \in X^{*}$ and $z \in Z$, we get, for any $T \in \mathscr{L}(Z, Y)$, that $\left(\Phi\left(x^{*} \otimes z\right)\right)(T)=\operatorname{trace}\left(T\left(\phi\left(x^{*} \otimes z\right)\right)\right)=\left(\phi x^{*}\right)(T z)=\left(\left(\phi x^{*}\right) \otimes z\right)(T)$. Hence

$$
\Phi\left(x^{*} \otimes z\right)=\left(\phi x^{*}\right) \otimes z, \quad x^{*} \in X^{*}, z \in Z,
$$

and therefore, for all $y^{*} \in Y^{*}$ and $z \in Z$,

$$
P\left(y^{*} \otimes z\right)=\Phi\left(\left(j^{*} y^{*}\right) \otimes z\right)=\left(\phi j^{*} y^{*}\right) \otimes z=\left(\pi y^{*}\right) \otimes z
$$

as desired.

COROLlaRY 4.7. Let $X$ be an ideal in a Banach space $Y$. If $X^{*}$ has the compact approximation property with conjugate operators, then $\mathscr{H}(Z, X)$ is an ideal in $\mathscr{W}(Z, Y)$ (and therefore also in $\mathscr{K}(Z, Y)$ ) for all Banach spaces $Z$.

PROOF. The proof is immediate from Theorem 4.6 and Theorem 4.4.

REMARK 4.2. Example 1.2 shows that the assumption ' $X$ * has the compact approximation property with conjugate operators' is essential in Corollary 4.7 and Theorem 4.6 (recall that $X$ is always an ideal in $X^{* *}$ ) and cannot be replaced by the assumption ' $X^{*}$ has the metric compact approximation property'.

COROLlaRY 4.8. Let $X$ be an $M$-ideal (respectively, a u-ideal having the unique ideal property) in a Banach space $Y$. If $X^{*}$ has the compact approximation property with conjugate operators, then $\mathscr{K}(Z, X)$ is an $M$-ideal (respectively, a u-ideal) in $\mathscr{K}(Z, Y)$ for all Banach spaces $Z$.

Proof. By Corollary $4.7, \mathscr{K}(Z, X)$ is an ideal in $\mathscr{K}(Z, Y)$ for all Banach spaces $Z$ and therefore Corollary 4.5 applies to obtain the desired conclusion.

REMARK 4.3. The assumption ' $X$ ' has the compact approximation property with conjugate operators' is also essential in Corollary 4.8 (see Remark 4.2). Namely, if $X$ is the closed subspace of $c_{0}$ constructed by Johnson and Schechtman (see [13, Corollary JS]), then $X$ is an $M$-ideal in $X^{* *}, X$ has a basis, and $X^{*}$ does not have the approximation property. Moreover, as it will be shown in a forthcoming paper of the authors, based on the present article and [19], there exists a separable reflexive Banach space $Z$ such that $\mathscr{K}(Z, X)$ is not an ideal in $\mathscr{X}\left(Z, X^{* *}\right)$. 


\section{References}

[1] E. M. Alfsen and E. G. Effros, 'Structure in real Banach spaces. Part I and II', Ann. of Math. 96 (1972), 98-173.

[2] C. J. Cabello, E. Nieto and E. Oja, 'On ideals of compact operators satisfying the $M(r, s)$ inequality', J. Math. Anal. Appl. 220 (1998), 334-348.

[3] P. G. Casazza and H. Jarchow, 'Self-induced compactness in Banach spaces', Proc. Royal Soc. Edinburgh Sect. A 126 (1996), 355-362.

[4] P. G. Casazza and N. J. Kalton, 'Notes on approximation properties in separable Banach spaces', in: Geometry of Banach Spaces, Proc. Conf. Strobl 1989 (eds. P. F. X. Müller and W. Schachermayer), London Math. Soc. Lecture Note Series 158 (Cambridge University Press, 1990) pp. 49-63.

[5] W. J. Davis, T. Figiel, W. B. Johnson and A. Pelczyński, 'Factoring weakly compact operators', J. Funct. Analysis 17 (1974), 311-327.

[6] J. Diestel and J. J. Uhl, Jr., Vector measures, Mathematical Surveys 15 (Amer. Math. Soc., Providence, RI, 1977).

[7] M. Feder and P. D. Saphar, 'Spaces of compact operators and their dual spaces', Israel J. Math. 21 (1975), 38-49.

[8] G. Godefroy, N. J. Kalton and P. D. Saphar, 'Unconditional ideals in Banach spaces', Studia Math. 104 (1993), 13-59.

[9] G. Godefroy and P. D. Saphar, 'Normes lisses et propriété d'approximation métrique', C. R. Acad. Sci. Paris, Sér. I 299 (1984), 753-756.

[10] _ ' 'Duality in spaces of operators and smooth norms on Banach spaces', Illinois J. Math. 32 (1988), 672-695.

[11] P. Harmand, D. Werner and W. Werner, $M$-ideals in Banach spaces and Banach algebras, Lecture Notes in Math. 1547 (Springer, Berlin, 1993).

[12] J. Johnson, 'Remarks on Banach spaces of compact operators', J. Funct. Analysis 32 (1979), 304-311.

[13] W. B. Johnson and T. Oikhberg, 'Separable lifting property and extensions of local reflexivity', Illinois J. Math. 45 (2001), 123-137.

[14] $\AA$. Lima, 'Intersection properties of balls and subspaces in Banach spaces', Trans. Amer. Math. Soc. 227 (1977), 1-62.

[15] — - 'The metric approximation property, norm-one projections and intersection properties of balls', Israel J. Math. 84 (1993), 451-475.

[16] _ - 'Property $\left(w M^{*}\right)$ and the unconditional metric compact approximation property', Studia Math. 113 (1995), 249-263.

[17] $\AA$. Lima, O. Nygaard and E. Oja, 'Isometric factorization of weakly compact operators and the approximation property', Israel J. Math. 119 (2000), 325-348.

[18] $\AA$. Lima and E. Oja, 'Ideals of finite rank operators, intersection properties of balls, and the approximation property', Studia Math. 133 (1999), 175-186.

[19] —_, 'Hahn-Banach extension operators and spaces of operators', Proc. Amer. Math. Soc. 130 (2002), 3631-3640.

[20] A. Lima, E. Oja, T. S. S. R. K. Rao and D. Werner, 'Geometry of operator spaces', Michigan Math. J. 41 (1994), 473-490.

[21] J. Lindenstrauss and L. Tzafriri, Classical Banach spaces I, Ergebnisse der Mathematik und ihrer Grenzgebiete 92 (Springer, 1977).

[22] E. Oja, ' $H B$-subspaces and Godun sets of subspaces in Banach spaces', Mathematika 44 (1997), 120-132.

[23] _ _ 'Géométrie des espaces de Banach ayant des approximations de l'identité contractantes', C. R. Acad. Sci. Paris, Sér. I 328 (1999), 1167-1170. 
[24] - 'Geometry of Banach spaces having shrinking approximations of the identity', Trans. Amer. Math. Soc. 352 (2000), 2801-2823.

[25] E. Oja and M. Põldvere, 'On subspaces of Banach spaces where every functional has a unique norm-preserving extension', Studia Math. 117 (1996), 289-306.

[26] _ - 'Intersection properties of ball sequences and uniqueness of Hahn-Banach extensions', Proc. Royal Soc. Edinburgh 129A (1999), 1251-1262.

[27] R. R. Phelps, 'Uniqueness of Hahn-Banach extensions and unique best approximation', Trans. Amer. Math. Soc. 95 (1960), 238-255.

[28] T. S. S. R. K. Rao, 'On ideals in Banach spaces', Rocky Mountain J. Math. 31 (2001), 595-609.

[29] B. Sims and D. Yost, 'Linear Hahn-Banach extension operators', Proc. Edinburgh Math. Soc. 32 (1989), 53-57.

[30] D. Werner, 'Denting points in tensor products of Banach spaces', Proc. Amer. Math. Soc. 101 (1987), 122-126.

[31] - ' $M$-structure in tensor products of Banach spaces', Math. Scand. 61 (1987), 149-164.

[32] G. Willis, 'The compact approximation property does not imply the approximation property', Studia Math. 103 (1992), 99-108.

Department of Mathematics

Agder College

Gimlemoen 25J

Serviceboks 422

4604 Kristiansand

Norway

e-mail: asvald.lima@hia.no
Faculty of Mathematics Tartu University Liivi 2-606 EE-50409 Tartu

Estonia

e-mail: eveoja@math.ut.ee 